\title{
Role of diffusion weighted MRI in assessment of hypervascular hepatic tumors
}

\author{
Amany M. Abd El-Aziz, Walid R. Abdelaziz, Tarek W. Hemada, Mariam E. Mosaad \\ Department of Radiodiagnosis,Faculty of Medicine - Ain Shams University \\ Corresponding author: Mariam E Mosaad; Mobile: 01006228978; Email:mariam.elsaid10@gmail.com
}

\begin{abstract}
Background: Hypervascular hepatic focal lesions may be benign or malignant. Therapy requires an accurate diagnosis, which in turn relies primarily on appropriate imaging and image-guided biopsy. MRI having many sequences, markedly helps in the detection of small lesions and in reaching the diagnosis easily even without contrast injection or the need for biopsy as in hemangiomas. Aim of the Work: To assess the role of diffusion weighted MRI imaging in differentiation between various types of hypervascular hepatic tumors. Patients $\boldsymbol{\&}$ Methods: This study included (24) patients, 12 males \& 12 females, their ages ranges from 28 to 66 years with a mean age of 49.5 years. The study was performed in Radiology department at Ain Shams University Hospitals and some private clinics in addition to National Cancer institute. Results: Highly statistically significant difference between benign and malignant according to ADC value. Benign lesions have higher mean ADC value compared to malignant lesions. Conclusion: Diffusion-weighted MRI sequence with quantitative ADC measurements can be useful in the differentiation of benign and malignant hypervascular hepatic focal lesions. Recommendations: Further studies on a larger scale of patients are needed to confirm the results obtain by this study.
\end{abstract}

Key words: DW MRI, hypervascular hepatic tumors

\section{INTRODUCTION}

Hypervascular hepatocellular lesions include both benign and malignant etiologies. In the benign category, focal nodular hyperplasia and adenoma are typically hypervascular. Malignant hypervascular primary hepatocellular lesions include hepatocellular carcinoma. Also certain cancers such as metastatic neuroendocrine tumors tend to produce hypervascular metastases due to the greater recruitment of arterial blood supply ${ }^{(1)}$.

Hepatocellular carcinoma (HCC) is the second leading cause of cancer-related death worldwide (2) with an estimated 500,000 to 1 million deaths per year ${ }^{(3)}$.

Ultrasound is frequently the first-line imaging modality for the study of the liver because of a combination of high spatial resolution and inherent soft tissue contrast, lack of ionizing radiation, low cost, and wide availability. Operator dependency, substantial image degradation in obese patients, and limited field of view, however, represent major limitations of this modality compared with other cross-sectional imaging techniques ${ }^{(4)}$.

MRI proved to be superior to $\mathrm{CT}$ in the detection of HCC and for the characterization of nodules in patients with liver cirrhosis because of the high tissue contrast provided by MRI ${ }^{(5)}$. Nowadays, it plays a key role in management of liver lesions, using a radiation-free technique and a safe contrast agent profile ${ }^{(6)}$.

Diffusion-weighted imaging (DWI) is a relatively recent technological improvement of MRI.
It allows the mapping of the free diffusion of water molecules (Brownian motions) which reflects the structural differences in disease by restricting diffusion ${ }^{(7)}$. Diffusion is expressed in an apparent diffusion coefficient (ADC), which reflects the diffusion properties unique to each type of tissue ${ }^{(5)}$.

DWI can be added to the routine examination easily using recently available machines. This imaging method has a good ability to detect liver lesions, and quantitative evaluation can be achieved without contrast media ${ }^{(7)}$.

The clinical uses of liver DW-MRI include improved: 1 . detection of focal liver lesions, 2. contribution to tissue characterization for both diffuse disease and focal lesions, 3 . monitoring of tumor response after chemotherapy or radiotherapy, 4.detection of recurrent disease, 5. differentiating recurrence from post-therapeutic change, and 6.potentially predicting treatment outcome ${ }^{(8)}$.

\section{AIM OF THE WORK}

The aim of the current study was to assess the role of diffusion weighted MRI imaging in differentiation between various types of hypervascular hepatic tumors.

\section{PATIENTS AND METHODS}

\section{Patients:}

This study included 24 patients, 12 males \& 12 females, their ages ranged from 28 to 66 years with a mean age of 49.5 years. 
The study was performed in Radiology department at Ain Shams University Hospitals and some private clinics in addition to National Cancer institute. The study was approved by the Ethics Board of Ain Shams University and an informed written consent was taken from each participant in the study.

Selection criteria: Patient known to have hypervascular hepatic focal lesions were detected by US and/or MSCT.

Exclusion criteria: Patients known to have contraindications for MRI e.g. an implanted magnetic device and pacemakers.

Patients were subjected to the following: Full clinical assessment including; recording of age, sex and clinical presentation. Laboratory investigations \{liver biochemical profile, renal function tests, Alpha fetoprotein $\}$. Abdominal MRI (pre- and post contrast study and diffusionweighted imaging).The results were compared to laboratory, histopathology (if available) and other previous radiological (US\&/or MSCT) findings done for all patients.

Abdominal MRI techniques: Conventional MRI, post Gd- DTPA dynamic and diffusion MR imaging were performed. First blind characterization and detection of focal lesions was performed, second the diffusion images with ADC values were reviewed. Third characterizing the enhancement pattern of the focal lesions was detected. Then correlation with laboratory finding and other imaging for the patient were done. MR imaging was performed on high field system (1.5 Tesla) magnet units (Philips Intera and Achieva) using a phased array coil to cover the whole liver.

\section{MR protocol used:}

a- Pre-contrast imaging included: T1
weighted $(\mathrm{TR})=10 \mathrm{msec}$, echo time $(\mathrm{TE})=4.58 \mathrm{msec}$, matrix 179/320, slice thickness 7-8mm, slice gap 1-2 mm, and FOV: $355 \mathrm{~mm}$. T2 weighted (T2W) images (single shot free breathing): $\mathrm{TR} \geq 445 \mathrm{~ms}, \mathrm{TE}=26-28$ msec, matrix 180-200x240 with a field of view: 365 , slice thickness 7-8mm, slice gap 1-2mm. T2 SPAIR (Spectral Attenuated Inversion Recovery) fat suppression sequence: TR $\geq 400 \mathrm{msec}$, TE $=80 \mathrm{msec}$, matrix $204 \times 384$ with a field of view: 365 , slice thickness 7-8mm, slice gap 1-2mm. In phase and out phase gradient echo sequence (Dual/FFE): $\mathrm{TR}=75$ $100 \mathrm{msec}, \mathrm{TE}=4.6 \mathrm{msec}$ for in phase and $2.3 \mathrm{msec}$ for out phase, matrix $143 \times 240$ with a field of view: 345 , slice thickness 7-8mm, slice gap $0 \mathrm{~mm}$. Heavy T2 weighted images: TR $=520 \mathrm{msec}, \quad \mathrm{TE}=200 \mathrm{msec}$, matrix 235/384 with a field of view: 375 , slice thickness 7-8mm, slice gap 1-2mm.

b- Dynamic study: Dynamic study was performed after bolus injection of $0.1 \mathrm{mmol} / \mathrm{kg}$ body weight of Gd-DTPA at a rate of $2 \mathrm{ml} / \mathrm{s}$, flushed with $20 \mathrm{ml}$ of sterile $0.9 \%$ saline solution from the antecubital vein. The injection of contrast media and saline solution was performed manually. Dynamic imaging using T1 THRIVE (High Resolution Isotropic Volume Examination) technique was performed in triphasic way [arterial phase (16-20 sec.), porto-venous phase $(45-60 \mathrm{sec}$.) and delayed equilibrium phase (3-5 min.) after administration of contrast media.

d- Diffusion study: Respiratory-triggered fatsuppressed single-shot echoplanar DW imaging was performed in the transverse plane with tri-directional diffusion gradients by using $\mathrm{b}$ values $0,500 \& 1000$ $\mathrm{sec} / \mathrm{mm}^{2}$ to increase sensitivity to cellular packing. Parallel imaging with generalized auto- calibrating partially parallel acquisition (GRAPPA) with an acceleration factor of two was applied to improve image quality. The other parameters were as follows: repetition time (TR) $\geq 1880 \mathrm{msec}$, echo time (TE) $=70 \mathrm{msec}$, number of excitations $(\mathrm{NEX})=3$, matrix $256 \times 256$ with a field of view as small as possible with $52 \%$ rectangular field of view, slice thickness 7-8mm, slice gap 1-2mm, scan time 3-4min.

\section{Imaging evaluation:}

The morphological features of each lesion were recorded included size, shape, margin, signal characteristics, pattern of enhancement in the dynamic imaging as well as number and site of the detected focal lesions. Then provisional diagnosis was reported. Second, we reviewed the diffusion images with ADC values for final radiological characterization and detection of focal lesions.

Sometimes we needed post processing image subtraction obtained between the post contrast imaging and pre-contrast images (in the same axial plane), using the software subtraction function available on the work station to evaluate the true contrast uptake in the post contrast image of the lesions appearing with high signal intensity in the pre contrast image. The results were compared and correlated with other radiological and laboratory results in all patients. 


\section{ADC calculation:}

The mean ADC of each focal lesion detected is measured by drawing a region of interest over the lesion. The ADC was measured twice and the two measurements were averaged. To ensure that the same areas were measured, regions of interest were copied and pasted from DW images to ADC maps.

\section{Statistical analysis:}

Recorded data were analyzed using the statistical package for social sciences, version 20.0 (SPSS Inc., Chicago, Illinois, USA). Quantitative data were expressed as mean \pm standard deviation (SD). Qualitative data were expressed as frequency and percentage.

The following tests were done: Chi-square $\left(x^{2}\right)$ test of significance was used in order to compare proportions between two qualitative parameters. The confidence interval was set to $95 \%$ and the margin of error accepted was set to $5 \%$. So, the p-value was considered significant as the following: Probability (P-value): P-value $<0.05$ was considered significant. $\mathrm{P}$-value $<0.001$ was considered as highly significant. $\mathrm{P}$-value $>0.05$ was considered insignificant.

\section{RESULTS}

There were about 119 arterially enhancing hypervascular focal lesions detected in all 24 patients. 43 lesions (in 8 patients) were diagnosed as HCC by typical imaging criteria in accordance with the American association of the study of liver disease (AASLD). 10 lesions were diagnosed as high grade dysplastic nodules in 3 patients. 27 lesions were diagnosed as cholangiocarcinoma in 2 patients. 12 lesions detected in 2 patients and were diagnosed as metastatic nodules. 3 lesions were diagnosed as FNH in 3 patients. 22 hemangioma lesions were detected in 7 patients. 2 adenomas were detected in 2 patients.

Table (2): Number and percentage of lesions in our study group patients.

\begin{tabular}{|l|c|c|}
\hline \multicolumn{1}{|c|}{ Type of the lesion } & $\begin{array}{c}\text { Number of } \\
\text { patients } \\
(\mathbf{N = 2 4 )}\end{array}$ & $\begin{array}{c}\text { Number of } \\
\text { lesions } \\
(\mathbf{N = 1 1 9})\end{array}$ \\
\hline Hepatocellular carcinoma (HCC) & $8(33.3 \%)$ & $43(36.1 \%)$ \\
\hline High grade dysplastic nodules (HGDN) & $3(12.5 \%)$ & $10(8.4 \%)$ \\
\hline Hemangioma & $7(29.2 \%)$ & $22(18.5 \%)$ \\
\hline Cholangiocarcinoma & $2(8.3 \%)$ & $27(22.7 \%)$ \\
\hline Metastatic nodules & $2(8.3 \%)$ & $12(10.1 \%)$ \\
\hline Focal nodular hyperplasia (FNH) & $3(12.5 \%)$ & $3(2.5 \%)$ \\
\hline Adenoma & $2(8.3 \%)$ & $2(1.7 \%)$ \\
\hline
\end{tabular}

\section{ADC values:}

ADC values were obtained for all 119 focal hepatic lesions detected at consensus reading. The mean ADC value of the 27 benign lesions was $1.45 \pm 0.16 \times 10-3 \mathrm{~mm}^{2} / \mathrm{sec}$. ADC values of benign lesions were between $1.15 \times 10-3$ and $1.88 \pm$ $0.11 \times 10-3 \mathrm{~mm}^{2} / \mathrm{sec}$. The highest mean ADC value was for hemangiomas. Among the benign lesions, adenoma had the lowest ADC value.

The ADC values of the 92 malignant lesions were between $1.04 \pm 0.11$ and $1.08 \times 10$ $3 \mathrm{~mm}^{2} / \mathrm{sec}$, with a mean value of $1.08 \pm 0.13 \times 10$ $3 \mathrm{~mm}^{2} / \mathrm{sec}$. Among the malignant lesions, the lowest mean ADC value was for metastasis, while some of HCC had the highest value.

The difference between the mean ADC values of benign and malignant lesions were statistically significant $(\mathrm{P}<0.0001)$. No statistically significant differences in ADC values among the different benign lesions or among the different malignant lesions.

Table (8): DWI and ADC signal criteria and mean ADC values of different hypervascular hepatic focal lesions.

\begin{tabular}{|l|c|c|c|}
\hline \multicolumn{1}{|c|}{ Mesion sequence } & DWI & ADC & $\begin{array}{c}\text { Mean ADC } \\
\text { value (mm2/sec) }\end{array}$ \\
\hline Hemangioma & $\begin{array}{c}\text { High (T2 shine } \\
\text { through effect) }\end{array}$ & High & $1.88 \pm 0.11 \times 10-3$ \\
\hline $\begin{array}{l}\text { Focal nodular } \\
\text { hyperplasia (FNH) }\end{array}$ & High & Low & $1.31 \pm 0.20 \times 10-3$ \\
\hline Adenoma & High & Low & $1.15 \times 10-3$ \\
\hline Cholangiocarcinoma & High & Low & $1.06 \pm 0.16 \times 10-3$ \\
\hline $\begin{array}{l}\text { Hepatocellular } \\
\text { carcinoma (HCC) }\end{array}$ & High & Low & $1.08 \pm 0.15 \times 10-3$ \\
\hline $\begin{array}{l}\text { High grade } \\
\text { dysplastic nodules } \\
\text { HGDN) }\end{array}$ & $\begin{array}{c}\text { Iso- to high } \\
\text { intense signal }\end{array}$ & $\begin{array}{c}\text { Iso- } \\
\text { intense }\end{array}$ & $1.12 \pm 0.11 \times 10-3$ \\
\hline signal & High & Low & $1.04 \pm 0.11 \times 10-3$ \\
\hline
\end{tabular}

Table (9): Comparison between benign and malignant according to ADC value.

\begin{tabular}{|c|c|c|c|}
\hline Lesion & $\begin{array}{c}\text { Mean ADC } \\
\text { value } \\
(\mathrm{mm} 2 / \mathrm{sec}) \times 10- \\
3\end{array}$ & t-test & p-value \\
\hline Benign & $1.45 \pm 0.16$ & \multirow{2}{*}{6.951} & \multirow{2}{*}{$<0.001$} \\
\hline Malignant & $1.08 \pm 0.13$ & & \\
\hline
\end{tabular}

This table shows highly statistically significant difference between benign and malignant according to ADC value. Benign lesions have higher mean ADC value compared to malignant lesions. 


\section{DISCUSSION}

With the widespread of cross-sectional imaging, a growth in rate of incidentally detected focal liver lesions (FLL) has been observed. A reliable detection and characterization of FLL is critical for optimal patient management. The majority of FLL arising in non cirrhotic liver are benign, even in patients with known extra-hepatic malignancy. Cysts, hemangiomas, focal nodular hyperplasias (FNH) and hepatocellular adenomas (HCA) are the most commonly encountered benign lesions ${ }^{(9)}$.

The most commonly encountered malignant lesions in noncirrhotic liver are metastases. Hepatocellular carcinomas (HCC), and to a lesser extent intrahepatic cholangiocarcinomas (IHC), occur mainly in the setting of chronic liver disease, and represent the most common primary liver malignancies ${ }^{(9)}$.

A tremendous development of new imaging techniques has taken place during these last years. Maximizing accuracy of imaging in the context of FLL is paramount in avoiding unnecessary biopsies. Nowadays, magnetic resonance plays a key role in management of liver lesions, using a radiation-free technique and a safe contrast agent profile ${ }^{(9)}$.

The amount of diffusion is defined using the diffusion coefficient. High cellularity, distortion of the extracellular space, and density of the hydrophobic cell membrane within the tissue restrict diffusion. In contrast, an intravoxelmicrovessel which travels disorderly behaves similarly to a diffusion phenomenon. DWI enables not only pure diffusion but also microvessel perfusion. Therefore, the diffusion coefficient is designated comprehensively as apparent diffusion coefficient (ADC) ${ }^{(7)}$.

The major aim of the present study is to determine the usefulness of diffusion weighted behavior in assessment of various hypervascular focal lesions of the liver, using ADC measurement, and to determine its contribution to differential diagnosis.

The current study was conducted including twenty four patients, 12 males and 12 females, with age ranging from 29-71 years and mean age of 54.6years, what means hepatic lesions are more predominant after age of 45-50 years, which was in line with other studies where Miller et al. (10) performed a study including 382 patients with age ranging from 15-88 years and mean age 59.2 years. Vergara et al. ${ }^{(11)}$ carried out a study including 26 patients with age ranging from 30-73 years and mean age 51.5 years.

DWI with high b-value, as in our study, mainly reflects diffusion information of water molecules motion within the lesions, which help to improve the characterization of solid FLLs. Meanwhile, we found in practice that DWI with higher b-value also enables a better detection of lesions in liver or pancreas compared with T2WI or other conventional sequences. For example, solid FLLs such as FNHs and hepatocellular carcinomas (HCCs) sometime can be difficult to be detected on T2WI or even DWI with low b-value due to either iso- or slightly hypersignal intensity to liver parenchyma; however, those lesions could be more conspicuous on DWI with higher b-value ${ }^{(\mathbf{1 2})}$.

In our study, hemangioma represents the commonest benign hepatic lesion as we had 22 out of 27 of benign FLLs.

In this study we had 7 cases of hemangiomas which displayed low signal on T1-WI, high signal on T2-WI \& maintained high signal on heavy T2-WI which agrees with Cogley and Miller ${ }^{(13)}$ who mentioned the same criteria in the pre-contrast study.

In our dynamic study, some hemangiomas lesions showed a nodular or "flame-shaped", discontinuous, peripheral enhancement was observed, as well as late, progressive, centripetal filling, and persistent delayed enhancement. Small hemangiomas $(<2 \mathrm{~cm})$ showed rapid complete filling on the late arterial phase images with no wash out. Enhancement criteria were in line with Matos et al., ${ }^{(9)}$ study.

On DWI, all 22 lesions showed signal intensity loss with increasing b-values \& showing high signal intensity and high value on ADC maps denoting benign nature of these lesions. On DWI images a T2 shine through effect may happen. T2 shine through is when T2 hyperintense fluid remains hyperintense on DWI. Shine through is due to high amount of water within the lesion; when a lot of water is present, there may be enough water incidentally not moving or not moving fast enough to have a significant decrease in signal intensity at lowto-medium $b$ values. This can be misleading. The shine through effect can be detected on ADC maps where areas of shine through on DWI will have high ADC values. Shine through effect can also be unveiled by increasing the $b$ value ${ }^{(14)}$. 
Regarding the practical implications, such DWI features of hepatic hemangiomas could be helpful in the differential diagnosis of focal hepatic lesions. For example, hypervascular solid tumors can be distinguished by their low ADCs, from the rapidly enhancing hemangiomas, showing high ADCs; meanwhile, centrally necrotic, cystic or mucinous metastatic tumors would show high ADCs and can be distinguished from the very slowly enhancing hemangiomas with low ADCs ${ }^{(15)}$.

\section{Focal nodular hyperplasia (FNH):}

In our study, we had 3 FNH lesions that showed isointense to hypointense signal on T1weighted images, on T2-weighted images, the lesion is slightly hyperintense. The central scar of FNH is hypointense on T1-weighted images, but on T2weighted images, the central scar appeared hyperintense.

In the dynamic study the lesions demonstrated marked, nearly uniform arterial-phase enhancement. The degree of lesion enhancement lessens on subsequent contrast-enhanced images, with lesion signal intensity approaching that of the surrounding liver parenchyma. The central scar has low signal intensity on early phase contrast-enhanced images but gradually enhances to become hyperintense relative to the rest of the lesion on delayed phase images which was in line with Campos et al. ${ }^{(16)}$ study.

\section{Hepatic adenoma:}

In our study, we had 2 cases of hepatic adenomas showing hypointense signal on T1weighted images, hyperintense signal on T2weighted images. Focal areas of fat may be seen as hypointense areas on $\mathrm{T} 1$ out-phased images due to signal drop.

In the dynamic study the lesions appeared as a hypervascular mass with persistent enhancement during dynamic evaluation which was in line with Grazioli et al. ${ }^{(17)}$ study.

\section{High grade dysplastic nodules:}

In our study, we had 3 cases of cirrhosisassociated dysplastic nodule that was of iso-intense signal in T1 \& T2WIs which agrees with ArifTiwari et al. (18) study who mentioned that dysplastic nodules typically show T2 signal that is similar to the background liver, there may be variable signal on T1-weighted images, similar to regenerative nodules.
On DWI, most high grade dysplastic nodules - which can be histologically difficult to separate out from a well-differentiated HCC- show increased signal intensity on DWI sequences which is in line with Niendorf et al. ${ }^{(19)}$ study.

\section{HCC:}

In our study, the majority of HCC lesions were hypointense on T1WIs, and few cases were isointense signals on T1WIs. The low T1 signal intensity pattern is typically characteristic for well differentiated HCC lesions. These findings were also noted by Arif-Tiwariet al. ${ }^{(\mathbf{1 8 )})}$ who reported that Focal HCC demonstrates variable signal on T1-weighted imaging, ranging from hyperintense to hypointense.

In our study, we noticed that $\mathrm{HCC}$ lesions (about 92\%) were hyperintense on T2WIs. This high T2 signal intensity pattern is typically characteristic for well differentiated HCC lesions. Arif-Tiwariet al. ${ }^{(\mathbf{1 8})}$ reported that elevated T2 signal in a focal lesion can be useful to reliably differentiate $\mathrm{HCC}$ from dysplastic nodules.

In our dynamic study, HCC lesions displayed the typical early arterial enhancement and contrast washout in the portal and delayed phases with persistent delayed enhancing outer rim "capsule". These findings were also similar to the publication of Shah et al. ${ }^{(20)}$.

In our study, HCC lesions showed evidence of restricted diffusion with high DW signal on increasing $b$-values that returned low signal on ADC maps denoting their malignant nature that was very useful in patients with no evidence of cirrhosis.

DWI nulls the signal within vessels, which is a significant difference, compared to $\mathrm{T} 2$ and $\mathrm{T} 1$ weighted images and probably the reason why small lesions adjacent to vessels are more easily detected on DWI. DWI increases the detection rate of HCC, particularly true for small cancers $<1 \mathrm{~cm}^{(\mathbf{1 4})}$.

\section{Cholangiocarcinoma:}

In our study, we had 2 cases of pathologically proven cholangiocarcinoma. It showed hypo- to isointense signal in T1WIs changing to hyperintense T2 signal with intrahepatic biliary dilatation which is line with Fábrega-Foster et al. ${ }^{(21)}$ study that show that on T1-weighted images, mass forming CCA presents as a hypo- to isointense mass. It may be mild to moderately hyperintense on T2-weighted images, 
depending on the relative proportions of central fibrous stroma vs. extracellular mucin.

On dynamic contrast enhanced MRI; it showed initial peripheral enhancement with concentric internal filling on delayed phase which is in line with Fábrega-Foster et al. ${ }^{(21)}$ study who showed that the most common pattern is a thin peripheral rim of enhancement in early arterial phase with progressive centripetal enhancement on the delayed phase.

On DWI, it showed increased signal intensity (restricted diffusion) \& lower ADC value.

\section{Metastatic deposits:}

The diagnosis of hypervascular metastatic lesions was more or less easier by knowing that the patients have primary malignancy, so this raised the importance of searching for a primary malignancy in cases we find multiple hepatic nodular lesions even in cirrhotic patients.

We have 2 cases with hypervascular metastatic deposits. Four metastatic lesions at a case of gastric intestinal stromal tumor (GIST) \& eight lesions at a case of gastric neuroendocrinal tumor(NET).

In our study we found that metastases appeared hypointense on T1WI \& hyperintense on T2WIs. Khosa et al. ${ }^{(22)}$ described similar appearance in their study where unenhanced T1-weighted images, hypervascular metastases generally were moderately hypointense relative to normal liver and on T2-weighted images, hypervascular metastases were usually hyperintense and may be cystic or necrotic.

In our dynamic study the hypervascular metastatic lesions displaying homogenous enhancement at the arterial phase with rapid wash out which is in line with Khosa et al. ${ }^{(22)}$ study, in which there was avid enhancement of lesions in arterial phase image with washout in the delayed phases and lesions became hypointense compared with background liver.

In our study, hypervascular metastatic lesions showed evidence of restricted diffusion on increasing b-values in DWI with low ADC signal.

\section{To what extent can DWI be useful in assessment of different hypervascular hepatic lesions?}

In our study, small lesions were effectively detected on diffusion weighted images, where lesions measuring $<1 \mathrm{~cm}$ were clearly depicted, thus making DW MRI a useful tool for detection of small focal hepatic lesions even without contrast injection.

A general assumption is that ADC values are higher in benign lesions and lower in malignant liver lesions. In fact, studies have found statistically significant difference in ADC values between benign and malignant liver lesions and there is a high degree of overlap between solid benign and malignant lesions ${ }^{(23)}$.

In our study, the mean ADC values were significantly different within benign hepatic focal lesions ( $\mathrm{P}<0.001)$ which agrees with Tokgoz et al. (17) study results. However, there was significant differences in ADC values among different malignant focal lesions in our study.

Using visual assessment of source DW-MR images performed using $\mathrm{b} 0$ and $\mathrm{b} 800$ allows detection and differentiation of focal liver lesions. All focal liver lesions showed hyperintensity on the b0 images, benign lesions tended to become isointense or hypointense on b800 images, and malignant lesions remained hyperintense. Quantitative ADC measurements can be used to aid focal liver lesion characterization, but it may be difficult to confidently describe a lesion as benign or malignant based on $\mathrm{ADC}$ values alone, due to the considerable overlap between groups of hepatic pathologies ${ }^{(8)}$.

In our study, there is similar ADC values between hepatic adenomas and high grade dysplastic nodules, similar results was in Albiin ${ }^{(24)}$ study who mentioned, benign solid hepatic lesions, such as focal nodular hyperplasia and adenomas, were found to have similar ADC values compared with malignant disease, such as metastases and HCC.

DWI is not specific and the restriction of water molecules can indicate a benign or malignant lesion. Small hemangiomas may restrict diffusion and be mistaken for malignancy, although hemangiomas are often markedly, diffusely T2 hyperintense and usually do not pose a diagnostic dilemma. Restricted diffusion is commonly seen in malignancy and is not specific to $\mathrm{HCC}$, as it is also seen in intrahepatic cholangiocarcinomas and hepatic metastases. The presence of hyperenhancement in the arterial phase and washout in the portal venous phase allow for the specific diagnosis of $\mathrm{HCC}^{(\mathbf{1 4})}$.

Some HCC do not restrict diffusion or only minimally restrict diffusion, particularly lesions 
with well differentiated components; this is possibly due to minimal difference in the cellularity of these well differentiated HCCs and the background liver parenchyma. Another cause for false negative DWI is cirrhosis. Cirrhotic hepatic parenchyma causes restricted diffusion of water molecules compared to normal parenchyma. In a fibrotic liver, a HCC will have decreased lesion to liver contrast on DW. Therefore, if a lesion is suspicious for HCC on conventional MR sequences, the absence of restricted diffusion does not necessarily exclude $\mathrm{HCC}$, especially if the background parenchyma is fibrotic ${ }^{(\mathbf{1 4})}$.

In our experience the radiologist should use all sequences to try to characterize lesions detected on $\mathrm{DWI}^{(\mathbf{1 4 )}}$.

\section{Limitations of DWI:}

Diffusion imaging has several limitations, mostly attributable to the EPI based nature of the sequence. SS EPI provides a limited image quality with low spatial resolution and poor SNR and is susceptible to several artifacts, including blurring, ghosting and distortions. Although modern scanners with multichannel coils, strong gradients, high magnetic fields and advanced software have been successful in reducing such effects to a great extent. In addition, parallel imaging techniques improve SNR by allowing a decrease in acquisition time (TE) ${ }^{(23)}$.

Our study had some limitations: The DW data set included was only respiratorytriggered images that have superiority over breathhold DW imaging for lesion detection, yet there are some limitations of the respiratory triggered technique like cardiac motion artifacts and noise contamination that may distort ADC values to a certain degree. Additional pulse triggering may overcome cardiac motion related artifacts. The low number of lesions, especially the benign solid hepatocellular lesions (e.g., hepatic adenoma, focal nodular hyperplasia), thus making the comparison between solid benign and malignant masses limited.

\section{CONCLUSION}

In our study we concluded that diffusionweighted MRI sequence with quantitative ADC measurements can be useful in the differentiation of benign and malignant hypervascular hepatic focal lesions. Both qualitative evaluation of high b- value DW-MR images and quantitative evaluation of ADC maps are employed for lesion characterization. The ADC values of benign lesions are significantly higher than those of malignant lesions, with variable degrees of overlap between the pathological entities. DWI proved to have value in the assessment of hypervascular hepatic focal liver lesions especially in detection of small lesions that are not clearly depicted by conventional sequences, but should always be used in conjunction with traditional MRI since there is great overlap between ADC values of benign and malignant lesions. It seems reasonable to use DWI in conjunction to conventional imaging for proper radiological characterization of the hypervascular hepatic focal lesions.

\section{REFERENCES}

1- Kamaya A, Maturen KE, Tye GA, Liu YI, Parti NN and Desser TS (2009): Hypervascular liver lesions. Semin Ultrasound CT MR., 30(5):387-407.

2- Torre LA, Bray F, Siegel RL, Ferlay J, Lortet-Tieulent $J$ and Jemal A (2015): Global cancer statistics, 2012. CA Cancer J Clin., 65:87-108.

3- Forner A, Llovet JMand Bruix J (2012): Hepatocellular carcinoma. Lancet, 379: 1245-55.

4- Marin D,Furlan A, Federle M, MidiriM and Brancatelli $G$ (2009): Imaging Approach for Evaluation of Focal Liver Lesions. Clinical gastroenterology and hepatology journal,7:624-634.

5- Kele $P$ and van der Jagt $E$ (2010): Diffusion weighted imaging in the liver. World J Gastroenterol., 16(13): 1567-1576.

6- Lee YJ, Lee JM, Lee JS, Lee HY, Park BH, Kim YH, Han JK and Choi BI (2015): Hepatocellular carcinoma: diagnostic performance of multidetector CT and MR imaging-a systematic review and metaanalysis. Radiology, 275:97-109.

7- $\quad$ Saito K, Tajima Y and Harada T (2016): Diffusion-weighted imaging of the liver: Current applications. World J Radiol., 8(11): 857-867. 
8- Bharwani N and Kohb D (2013): Diffusion-weighted imaging of the liver: an update Cancer Imaging, 13(2): 171-185.

9- Matos AP, Velloni F, Ramalho M, AlObaidy M, Rajapaksha A and Semelka R (2015): Focal liver lesions: Practical magnetic resonance imaging approach World J Hepatol., 7(16): 1987-2008.

10- Miller FH, Hammond N, Siddiqi AJ, Shroff S, Khatri G, Wang Y, Merrick LB and Nikolaidis $P$ (2010): Utility of Diffusion-Weighted MRI in Distinguishing Benign and Malignant Hepatic Lesions. Journal Of Magnetic Resonance Imaging, 32:138-147.

11- Vergara ML, Fernández $M$ and Pereira $R$ (2010): Diffusion-weighted MRI characterization of solid liver lesions. Revista Chilena De Radiología, 16 (1): 510.

12- Yang D, Wang $K$, Yao $X$, Ye H, Jiang T, Liu Y, Gao J, Chen M, Zhou C and Yang Z (2016): Diffusion-Weighted Imaging with Two Different b-Values in Detection of Solid Focal Liver Lesions Biomed Res Int., 812881236.

13- Cogley JR and Miller FH (2014): MR imaging of benign focal liver lesions. RadiolClin North Am., 52(4):657-82.

14- Gluskin J, Chegai F, Monti S, SquillaciE and Mannelli $L$ (2016): Hepatocellular Carcinoma and Diffusion-Weighted MRI: Detection and Evaluation of Treatment Response J Cancer, 7(11):1565-1570.

15- Nam S, Park K, Yu J, Chung J, Kim J and Kim K (2012): Hepatic Cavernous Hemangiomas: Relationship between Speed of Intratumoral Enhancement during Dynamic MRI and Apparent Diffusion Coefficient on Diffusion-Weighted Imaging Korean J Radiol., 13(6): 728-735.

16- Campos J, Sirlin C and Choi J (2012): Focal hepatic lesions in Gd-EOB-DTPA enhanced MRI: the atlas Insights Imaging, 3(5): 451-474.

17- Grazioli L, Olivetti L, Mazza $G$ and PiaBondioni M (2013): MR Imaging of Hepatocellular Adenomas and Differential Diagnosis Dilemma Int J Hepatol., 2013: 374170.
18- Arif-Tiwari H, Kalb B, Chundru S, Sharma P, Costello J, Guessner $R$ and Martin D (2014): MRI of hepatocellular carcinoma: an update of current practices DiagnIntervRadiol., 20(3): 209-221.

19- Niendorf E, Spilseth $B$, Wang $X$ and Taylor A (2015): Contrast Enhanced MRI in the Diagnosis of HCC Diagnostics (Basel), 5(3): 383-398.

20- Shah S, Shukla A and Paunipagar B (2014): Radiological Features of Hepatocellular Carcinoma J Clin Exp Hepatol., 4(3): S63-S66.

21- Fábrega-Foster K, Ghasabeh M, Pawlik T and Kamel I (2017): Multimodality imaging of intrahepatic cholangiocarcinoma Hepatobiliary. Surg Nutr., 6(2): 67-78.

22- Khosa F, Khan AN andEisenberg RL (2011): Hypervascular liver lesions on MRI. AJR Am J Roentgenol., 197(2):W204-20.

23- Shenoy-Bhangle A, Baliyan V, Kordbacheh H, Guimaraes $A$ and Kambadakone A (2017): Diffusion weighted magnetic resonance imaging of liver: Principles, clinical applications and recent updates. World J Hepatol., 9(26): 1081-1091.

24- Albiin N (2012): MRI of Focal Liver Lesions. Curr Med Imaging Rev., 8(2): 107116. 